\title{
Identifying the incidence of rash, Stevens-Johnson syndrome and toxic epidermal necrolysis in patients taking lamotrigine: a systematic review of 122 randomized controlled trials*
}

\author{
Romi Bloom ${ }^{1}$
}

Kyle T. Amber ${ }^{2}$

DOI: http://dx.doi.org/10.1590/abd1806-4841.20175070

\begin{abstract}
Lamotrigine is an antiepileptic drug used for the treatment of epilepsy, bipolar disorder and numerous off-label uses. The development of rash significantly affects its use. The most concerning of these adverse reactions is Stevens-Johnson syndrome/toxic epidermal necrolysis. We performed a systematic review of randomized controlled trials using lamotrigine as a monotherapy to quantify the incidence of cutaneous reactions, particularly Stevens-Johnson syndrome/toxic epidermal necrolysis. Of a total of 4,364 papers regarding lamotrigine, 122 studies met our inclusion and exclusion criteria. In total, 18,698 patients were included with 1,570 (8.3\%) of patients experiencing an adverse dermatologic reaction. The incidence of StevensJohnson syndrome/toxic epidermal necrolysis was $0.04 \%$.
\end{abstract}

Keywords: Adverse drug reaction reporting systems; Drug eruptions; Drug Toxicity; Stevens-Johnson Syndrome

Lamotrigine is an antiepileptic drug used for the treatment of epilepsy, bipolar disorder and numerous off-label uses..-3 The development of rash significantly affects the use of this drug, as almost $10 \%$ of seizure patients treated with lamotrigine develop some form of rash. ${ }^{4}$ This occurs at a greater rate that than other antiepileptic drugs, particularly non-aromatic antiepileptic drugs. ${ }^{4}$ The most concerning of these adverse skin reactions is the increased risk of developing Stevens-Johnson syndrome or toxic epidermal necrolysis (SJS/TEN). ${ }^{5}$ The incidence of SJS/TEN has yet to be quantitated in a large cohort of patients taking lamotrigine.

While the exact causal relation remains unclear, certain HLA types have been associated with the development of SJS/ TEN following lamotrigine. Zeng at al. identified HLA-B*15:02 in the Han Chinese population. ${ }^{6}$ This was validated in a subsequent meta-analysis. ${ }^{7}$ Park et al. demonstrated an association between the HLA-B*44:03 allele and SJS/TEN in the Korean population. ${ }^{8}$
In order to assess the incidence of SJS/TEN with the use of lamotrigine as well as compare its incidence to other known causative drugs, we performed a systematic review of randomized controlled trials using lamotrigine as a monotherapy to quantify the incidence of cutaneous reactions, particularly SJS/TEN. We additionally sought to compare this incidence between different major disease indications: seizure disorder, bipolar disorder and other indications to evaluate whether an underlying epigenetic link in epileptic patients may make them more prone than bipolar patients to develop SJS with lamotrigine. This would potentially aid in further characterizing epigenetic links in SJS/TEN.

We performed a systematic review using Medline/PubMed database on May $3^{\text {rd }}, 2015$. Only English randomized controlled trials (RCTs) assessing lamotrigine as monotherapy were included. We defined monotherapy as instances where lamotrigine was used alone or as a sole addition to a pre-existing stable regimen.

Received on 24.11.2015

Approved by the Advisory Board and accepted for publication on 20.02.2016

* Study conducted at the University of California Irvine, Department of Dermatology - Irvine, California.

Financial Support: None.

Conflict of Interest: None.

Northshore University Health System, University of Chicago Pritzker School of Medicine - Chicago, Illinois.

University of California Irvine, Department of Dermatology - Irvine, California.

C2017 by Anais Brasileiros de Dermatologia 
TABLE 1: Incidence of adverse skin reactions and SJS/TEN in patients started on lamotrigine in randomized controlled trials

\begin{tabular}{llll}
\hline Patients treated with lamotrigine & \multicolumn{2}{l}{ Patients developing skin reactions } & \multicolumn{2}{l}{ SJS/TEN } \\
\hline Seizure N=6,805 & $534(7.8 \%)$ & $\mathrm{P}=0.119$ & $5(0.07 \%)$ \\
Bipolar N=9,947 & $864(8.6 \%)$ & & $2(0.02 \%)$ \\
Other N=1,946 & $172(8.8 \%)$ & $1(0.05 \%)$ \\
Total N=18,698 & $1,570(8.3 \%)$ & $8(0.04 \%)$ \\
\hline
\end{tabular}

SJS/TEN - Steven-Johnson syndrome/toxic epidermal necrolysis

This excluded studies where two or more drugs were started simultaneously. We used the search term lamotrigine and then filtered results to only randomized controlled trials. Abstracts were then reviewed to determine whether lamotrigine was used as a monotherapy. For each included paper, the total sample size, disease process being studied (seizures, bipolar disorder or other diseases), number of skin reactions and type of skin reactions were recorded. The incidence of skin reactions was calculated for each disease process as well as the total incidence. To calculate this, we divided the total number of patients developing all skin reactions or just SJS/ TEN by the total number of patients in the group being studied (i.e. each individual disease group and the total lamotrigine cohort). We additionally used a $\chi^{2}$ test to assess heterogeneity of skin reactions between each primary disease process to evaluate whether certain underlying conditions carried a greater risk of adverse cutaneous reactions using the SPSS 20 (IBM) software. Significance was set at $\mathrm{p}<0.05$ with a two-tailed test.

Of a total of 4,364 papers regarding lamotrigine, 255 randomized controlled trials were identified. Four of these articles were excluded, as they were not in English. After reviewing the abstracts and/or texts of these papers, 122 studies, ranging from 1993-2013, remained. A vast majority of excluded studies were those that initiated 2 or more new antiepileptics at once and those that did not report adverse events. In total, 18,698 patients were included with $1,570(8.3 \%)$ of patients experiencing an adverse dermatologic reaction. The incidence of SJS/TEN was $0.04 \%$. This translates to 1 patient per 2,500 treated. There was no significant variance in incidence amongst the different disease processes. Table 1 summarizes the incidences of skin reactions and SJS/TEN in particular for each disease process.
Adverse skin reactions occur in $8.3 \%$ of patients taking lamotrigine, with $0.04 \%$ of patients developing SJS/TEN. This rate of SJS/TEN is far greater than that reported for other well-known trigger medications including phenobarbital $(0.02 \%)$, nitrofurantoin $(0.007 \%)$ and sulfamethoxazole with trimethoprim $(0.003 \%) .{ }^{9}$ The incidence of skin reactions and SJS/TEN in particular did not demonstrate significant variance between seizure disorder, bipolar disorder and other indications. Thus, based on the current analysis, no disease cluster appears to be more susceptible to developing SJS/TEN. This analysis was, however, limited by using the general disease process rather than sub-types. The overall low incidence of SJS/TEN would however limit the statistical comparison of incidence rates, as identifying a statistically significant change in incidence with a disease.

Another limitation of this study is the anonymity of the assessors of the dermatological complications and the lack of clarity about how culpability was attributed. This is a limitation that prevented us from characterizing different rashes; thus, our endpoints were rash and SJS/TEN. By using only randomized controlled trials of lamotrigine, we minimized any possible publication biases by including case reports or retrospective manuscripts with emphasis on cutaneous adverse events. Cutaneous adverse reactions, particularly SJS/TEN, occur at a sizeable rate in patients receiving lamotrigine; herein we quantify this incidence and the homogeneity amongst different primary disease processes. We demonstrate that compared to the major culprit drugs phenobarbital, nitrofurantoin and sulfamethoxazole/trimethoprim, lamotrigine carries a higher incidence of SJS/TEN.] 


\section{REFERENCES}

1. Gamble CL, Williamson PR, Marson AG. Lamotrigine versus carbamazepine monotherapy for epilepsy. Cochrane Database Syst Rev. 2006;1:CD001031.

2. Reid JG, Gitlin MJ, Altshuler LL. Lamotrigine in psychiatric disorders. J Clin Psychiatry. 2013;74:675-84.

3. Reimers A. Trends and changes in the clinical use of lamotrigine. Pharmacoepidemiol Drug Saf. 2009;18:132-9.

4. Wang XQ, Xiong J, Xu WH, Yu SY, Huang XS, Zhang JT, et al. Risk of a lamotriginerelated skin rash: current meta-analysis and postmarketing cohort analysis. Seizure. 2015;25:52-61.

5. Mockenhaupt M, Viboud C, Dunant A, Naldi L, Halevy S, Bouwes Bavinck JN, et al. Stevens-Johnson syndrome and toxic epidermal necrolysis: assessment of medication risks with emphasis on recently marketed drugs. The EuroSCAR-study. J Invest Dermatol. 2008;128:35-44

6. Zeng T, Long YS, Min FL, Liao WP, Shi YW. Association of HLA-B*1502 allele with lamotrigine-induced Stevens-Johnson syndrome and toxic epidermal necrolysis in Han Chinese subjects: a meta-analysis. Int J Dermatol. 2015;54:488-93.

7. Li X, Yu K, Mei S, Huo J, Wang J, Zhu Y, et al. HLA-B*1502 increases the risk of phenytoin or lamotrigine induced Stevens-Johnson Syndrome/toxic epidermal necrolysis: evidence from a meta-analysis of nine case-control studies. Drug Res (Stuttg). 2015;65:107-11

8. Park HJ, Kim SR, Leem DW, Moon IJ, Koh BS, Park KH, et al. Clinical features of and genetic predisposition to drug-induced Stevens-Johnson syndrome and toxic epidermal necrolysis in a single Korean tertiary institution patients-investigating the relation between the HLA $-B^{\star} 4403$ allele and lamotrigine. Eur J Clin Pharmacol. 2015;71:35-41.

9. Chan HL, Stern RS, Arndt KA, Langlois J, Jick SS, Jick H, et al. The incidence of erythema multiforme, Stevens-Johnson syndrome, and toxic epidermal necrolysis. A population-based study with particular reference to reactions caused by drugs among outpatients. Arch Dermatol. 1990;126:43-7
MAILING ADDRESS:

Dr. Kyle T. Amber

118 Med Surg I.

Irvine, CA 92697

USA.

E-mail:kamber@uci.edu

How to cite this article: Bloom R, Amber KT. Identifying the incidence of rash, Stevens-Johnson syndrome and toxic epidermal necrolysis in patients taking lamotrigine: a Systematic review of 122 randomized controlled trials. An Bras Dermatol. 2017;92(1):139-41. 